\title{
Consistent Change Point Detection for Piecewise Constant Signals With Normalized Fused LASSO
}

\author{
Arash Owrang, Mohammadreza Malek-Mohammadi, Alexandre Proutiere and Magnus Jansson
}

\begin{abstract}
We consider the problem of offline change point detection from noisy piecewise constant signals. We propose normalized fused LASSO, an extension of the fused LASSO, obtained by normalizing the columns of the sensing matrix of the LASSO equivalent. We analyze the performance of the proposed method, and in particular, we show that it is consistent in detecting change points as the noise variance tends to zero. Numerical experiments support our theoretical findings.
\end{abstract}

Index Terms-Change point detection, piecewise constant, fused LASSO, irrepresentable condition.

\section{INTRODUCTION}

$\mathbf{P}$ IECEWISE constant signals play a major role in numerous applications such as comparative genomic hybridization [1], analysis of financial time-series [2], bio-medical imaging [3], smart power grids [4] and data segmentation. These applications call for detecting the change points from perturbed measurements. Numerous change point detection methods have been proposed [5]-[8]. In particular, the fused LASSO (FL) has attracted a lot of attention recently [7].

FL is closely related to the standard LASSO, and exploits the sparsity of piecewise constant signals in the discrete derivative domain [9]. Although FL provides a rather good match to the true piecewise constant signal, it can get cluttered with small additional steps [6], [10], [11]. In fact, it is shown that FL is inconsistent in detecting the true change points even asymptotically [6], [11]. More precisely, if two successive steps in the true piecewise constant signal are in the same direction, then FL most often detects spurious change points in addition to the true ones, even when the noise variance tends to zero. To overcome this issue, some methods propose to post-process the solution of FL [6], [10]. This obviously increases the computational cost. In an alternative approach, [12] proposes an iterative method based on solving a weighted LASSO in each iteration to suppress the spurious change points. Despite its superiority to FL in detecting the true change points, its performance is dependent on the distance between the true change points. Furthermore, this method appears computationally more expensive than FL.

In this paper, we consider the equivalent form of FL in the standard LASSO format. We prove that by normalizing the columns of the sensing matrix of the so-called LASSO equivalent, the irrepresentable condition [13] is satisfied. That is to say, this normalized FL (NFL) is asymptotically consistent in

This work was supported in part by the Swedish Research Council under contract 2015-05484.

The authors are with the ACCESS Linnaeus Centre, KTH Royal Institute of Technology, Stockholm, Sweden. detecting the true change points when the noise level converges to zero. Hence, if the amplitude of the smallest step in the true piecewise constant signal is reasonably larger than the standard deviation of the noise, NFL detects the change points accurately even with a limited number of measurements. We also emphasize that NFL and FL have the same computational complexity. We provide numerical examples that illustrate the superiority of NFL.

\section{FROM FUSED LASSO TO NORMALIZED FUSED LASSO}

A. Model

We have access to $N$ noisy measurements $\{y(t)\}_{t=1}^{N}$ of the true piecewise constant signal $m^{*}(t)$ according to the model

$$
y(t)=m^{*}(t)+\sigma \epsilon(t), \quad t \in\{1, \ldots, N\},
$$

where the additive random noise samples, $\epsilon(t)$ 's, are temporally independent and identically Gaussian distributed with $\epsilon(t) \sim \mathcal{N}(0,1)$ and $\sigma$ denotes the unknown standard deviation. The signal $m^{*}(t)$ is piecewise constant; i.e., there are $K$ unknown change points $\left\{s_{i}\right\}_{i=1}^{K}$ and $K+1$ values $\bar{m}_{1}, \ldots, \bar{m}_{K+1}$ such that for all $i=1, \ldots, K+1, m^{*}(t)=\bar{m}_{i}$ for all $s_{i-1}<t \leq s_{i}$ with the convention that $s_{0}=0$ and $s_{K+1}=N$. Moreover, we assume that $m^{*}(t)$ is constant for at least two consecutive samples, i.e.,

$$
s_{1} \geq 2, s_{i}-s_{i-1} \geq 2 \text { for } 1<i \leq K, s_{K} \leq N-2 .
$$

Our objective is to devise a method able to detect the change points from the noisy measurements $\{y(t)\}_{t=1}^{N}$. This method should be at least asymptotically consistent, meaning that when the noise level $\sigma$ tends to 0 , the method should detect the true change points.

\section{B. The Failure of the fused LASSO}

FL exploits the temporal sparsity of the discrete derivative of the piecewise constant signals using $\ell_{1}$ regularization [7]. FL aims at striking an appropriate trade-off between reducing the measurement noise and the piecewise constant structure by solving

$$
\hat{\mathbf{m}}^{\mathrm{FL}}=\underset{\mathbf{m} \in \mathbb{R}^{N}}{\operatorname{argmin}}\left\{\frac{1}{2}\|\mathbf{y}-\mathbf{m}\|_{2}^{2}+\lambda\|\mathbf{D m}\|_{1}\right\},
$$

where $\mathbf{y} \in \mathbb{R}^{N}$ is the measurement vector constructed as $\mathbf{y}=$ $[y(1), \ldots, y(N)]^{T}$, the matrix $\mathbf{D} \in \mathbb{R}^{(N-1) \times N}$ defined by

$$
\mathbf{D}=\left[\begin{array}{cccccc}
-1 & 1 & 0 & & \ldots & 0 \\
0 & -1 & 1 & 0 & \ldots & 0 \\
& & & & \ldots & \\
0 & \ldots & & 0 & -1 & 1
\end{array}\right]
$$




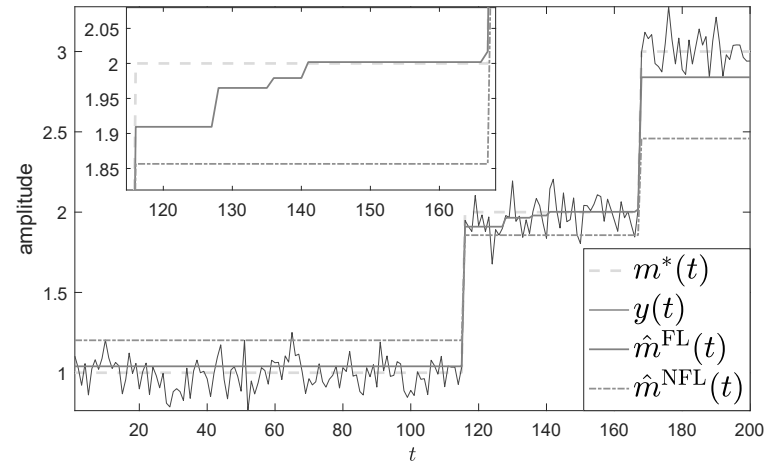

Fig. 1. The solution of FL, $\hat{m}^{\mathrm{FL}}(t)$, is cluttered with small steps when $\sigma=0.1$. The small box in the left top corner magnifies the intermediate level of $\hat{m}^{\mathrm{FL}}(t)$ and $\hat{m}^{\mathrm{NFL}}(t)(y(t)$ is eliminated for the sake of visibility).

acts as the difference operator and $\lambda>0$ is the regularization parameter, which implicitly controls the number of detected change points. Unfortunately, in a general setting for $m^{*}(t)$, FL fails at detecting the true change points. This is due to the fact that $\hat{\mathbf{m}}^{\mathrm{FL}}$ may be cluttered with small undesired steps forming a staircase [11]. Fig. 1 illustrates these undesired extra steps in the solution of FL. To understand the failure of FL, we adopt the equivalent of (3) in the standard LASSO format. LASSO has been extensively studied, and the literature provides sufficient conditions for perfect detection [13]-[15]. The standard LASSO reformulation of (3) has been introduced in [11] as

$$
\min _{\mathbf{x} \in \mathbb{R}^{N-1}}\left\{\frac{1}{2}\|\tilde{\mathbf{y}}-\mathbf{A x}\|_{2}^{2}+\lambda\|\mathbf{x}\|_{1}\right\}
$$

where $\tilde{\mathbf{y}}=\mathbf{y}-\frac{\mathbf{y}^{T} \mathbf{1}}{N} \mathbf{1}, \mathbf{1}=[1, \ldots, 1]^{T}$ and $\mathbf{A} \in \mathbb{R}^{N \times(N-1)}$ is given by

$$
a_{i, j}=\frac{j}{N}-1, \text { for } i \leq j \quad \text { and } \quad a_{i, j}=\frac{j}{N} \text {, otherwise. }
$$

This reformulation basically implies that the data model is transformed to

$$
\tilde{\mathbf{y}}=\mathbf{A} \mathbf{x}^{*}+\sigma \tilde{\boldsymbol{\epsilon}},
$$

where $\tilde{\boldsymbol{\epsilon}}$ is a random Gaussian perturbation with $\tilde{\boldsymbol{\epsilon}} \sim \mathcal{N}(\mathbf{0}, \mathbf{C})$, $\mathbf{C}$ is given by $c_{i i}=\frac{N-1}{N}, c_{i j}=-1 / N$ for $i \neq j$ and $\mathbf{x}^{*}=$ Dm* [11]. We know that LASSO can potentially select the true non-zero elements if the irrepresentable condition holds [13], [15]. The irrepresentable condition for problem (4) is

$$
\left\|\mathbf{B} \operatorname{sgn}\left(\mathbf{x}_{\mathcal{S}}^{*}\right)\right\|_{\infty}<1
$$

where $\operatorname{sgn}(\cdot)$ denotes the element-wise sign function and $\mathcal{S}$ denotes the ordered sequence of the true change points. Also, the vector $\mathrm{x}_{\mathcal{S}}^{*}$ is the collection of $x_{i}^{*}$ 's with the support $\mathcal{S}$, matrix $\mathbf{B}$ is defined as

$$
\mathbf{B}=\mathbf{A}_{\mathcal{S}^{c}}^{T} \mathbf{A}_{\mathcal{S}}\left(\mathbf{A}_{\mathcal{S}}^{T} \mathbf{A}_{\mathcal{S}}\right)^{-1}
$$

where $\mathbf{A}_{\mathcal{S}}$ is the collection of the columns of $\mathbf{A}$ with the support $\mathcal{S}$, and $\mathcal{S}^{c}$ represents the relative complement of $\mathcal{S}$ in $\{1, \ldots, N-1\}$. It is shown that $\mathbf{B}$ has a specific structure described by

$$
\mathbf{B}=\left[\begin{array}{llll}
\mathbf{B}^{1} & \mathbf{B}^{2} & \ldots & \mathbf{B}^{K+1}
\end{array}\right]^{T},
$$

where the columns of $\mathbf{B}^{r}$ 's (rows of $\mathbf{B}$ ) are defined as

$$
\begin{aligned}
& \mathbf{b}_{k}^{1}=\left[\frac{k}{s_{1}}, 0, \ldots, 0\right]^{T}, \\
& \mathbf{b}_{k}^{K+1}=\left[0, \ldots, 0,1-\frac{k}{N+1-s_{K}}\right]^{T}, 1 \leq k \leq N-s_{K} \\
& \mathbf{b}_{k}^{r}=[\underbrace{0, \ldots, 0}_{r-2}, 1-\frac{k}{s_{r}-s_{r-1}}, \frac{k}{s_{r}-s_{r-1}}, 0, \ldots, 0]_{1},
\end{aligned}
$$

for $2 \leq r \leq K$ and $1 \leq k \leq s_{r}-s_{r-1}-1$ [11]. Now it is clear that if $\operatorname{sgn}\left(x_{r-1}^{*}\right)=\operatorname{sgn}\left(x_{r}^{*}\right)$ for $r \in \mathcal{S}$, then $\mathbf{b}_{k}^{r T} \operatorname{sgn}\left(\mathbf{x}_{\mathcal{S}}^{*}\right)=1$ implying that the irrepresentable condition is not satisfied. This suggests that FL cannot perfectly detect the true change points when two consecutive steps are in the same direction. On the contrary, if every two consecutive steps are in the opposite direction, i.e., $\operatorname{sgn}\left(x_{r-1}^{*}\right)=-\operatorname{sgn}\left(x_{r}^{*}\right)$ for all $r$ 's, the irrepresentable condition is preserved.

\section{The Normalized Fused LASSO}

We know that the sensing matrix on which LASSO operates ideally should have unit-norm columns [14]. Otherwise, LASSO tends to select the parameters that are associated with columns having large norm. Now, in the LASSO reformulation (4), it is easy to see that the columns of $\tilde{\mathbf{A}}$ are not normalized. This motivates the introduction of the normalized FL as

$$
\hat{\mathbf{x}}^{\mathrm{NFL}}=\underset{\tilde{\mathbf{x}} \in \mathbb{R}^{N-1}}{\operatorname{argmin}}\left\{\frac{1}{2}\|\tilde{\mathbf{y}}-\tilde{\mathbf{A}} \tilde{\mathbf{x}}\|_{2}^{2}+\lambda\|\tilde{\mathbf{x}}\|_{1}\right\},
$$

where the columns of $\mathbf{A}$ are normalized as $\tilde{\mathbf{A}}=\mathbf{A} \mathbf{W}^{-1}$ and $\mathbf{W}$ is a diagonal matrix with diagonal elements $w_{i i}=$ $\sqrt{i(N-i) / N}$ for all $i$. Note that after normalizing the columns of $\mathbf{A}$, the model becomes $\tilde{\mathbf{y}}=\tilde{\mathbf{A}} \tilde{\mathbf{x}}^{*}+\sigma \tilde{\boldsymbol{\epsilon}}$ where $\tilde{\mathbf{x}}^{*}=\mathbf{W} \mathbf{x}^{*}$. Alternatively, the optimization problem (10) can be transformed back to the standard FL format as

$$
\min _{\mathbf{m} \in \mathbb{R}^{N}} \frac{1}{2}\|\mathbf{y}-\mathbf{m}\|_{2}^{2}+\lambda\|\mathbf{W D m}\|_{1} .
$$

To solve the above optimization problem, one can use the weighted taut-string algorithm [16], which has a computational complexity similar to that of the taut-string algorithm [17] for solving (3).

Remarkably, $\tilde{\mathbf{A}}$ now satisfies the irrepresentable condition as stated in the following lemma. This suggests that NFL could detect the true change points asymptotically accurately, even if consecutive steps in $m^{*}$ are in the same direction. This will be confirmed in the next section where we analyze the performance of NFL. Fig. 1 illustrates a clutter-free $\hat{m}^{\mathrm{NFL}}$ in contrast to the cluttered $\hat{m}^{\mathrm{FL}}$.

Lemma 1. Consider any $\mathcal{S}$ that satisfies (2). For any arbitrary $\operatorname{sgn}\left(\tilde{\mathbf{x}}_{\mathcal{S}}^{*}\right), \tilde{\mathbf{A}}_{\mathcal{S}}$ and $\operatorname{sgn}\left(\tilde{\mathbf{x}}_{\mathcal{S}}^{*}\right)$ satisfy the irrepresentable condition.

Proof. Using $\tilde{\mathbf{A}}$ to replace $\mathbf{A}$ in (8), we get the irrepresentable condition for problem (10) as

$$
\left\|\mathbf{W}_{\mathcal{S}^{c}, \mathcal{S}^{c}}^{-1} \mathbf{B} \mathbf{W}_{\mathcal{S}, \mathcal{S}} \operatorname{sgn}\left(\tilde{\mathbf{x}}_{\mathcal{S}}^{*}\right)\right\|_{\infty}<1,
$$


where $\mathbf{W}_{\mathcal{S}, \mathcal{S}}$ is a diagonal matrix formed by the columns and the rows of $\mathbf{W}$ with the support $\mathcal{S}$. Now by using basic properties of norms, we have

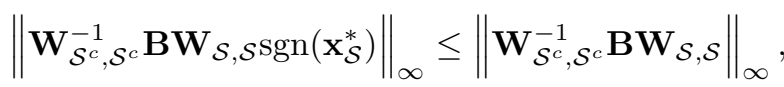

where the $\infty$-norm of a matrix is the largest absolute row sum. Exploiting (9) and the expression for $w_{i i}$, the absolute row sum of $\mathbf{W}_{\mathcal{S}^{c}, \mathcal{S}^{c}}^{-1} \mathbf{B} \mathbf{W}_{\mathcal{S}, \mathcal{S}}$ for $\mathbf{B}^{r}, r \neq\{1, K+1\}$ can be written as

$$
\left(1-\frac{k}{s_{r}-s_{r-1}}\right) \frac{g\left(s_{r-1}\right)}{g\left(s_{r-1}+k\right)}+\frac{k}{s_{r}-s_{r-1}} \frac{g\left(s_{r}\right)}{g\left(s_{r-1}+k\right)},
$$

where the function $g(\cdot)$ is defined as $g(a)=\sqrt{a(N-a)}$ for $0<a \leq N$. By a straightforward manipulation, it becomes

$$
\left[g\left(s_{r-1}\right)+\frac{\left[g\left(s_{r}\right)-g\left(s_{r-1}\right)\right] k}{s_{r}-s_{r-1}}\right] / g\left(s_{r-1}+k\right) .
$$

Note that the irrepresentable condition is trivially satisfied for the cases $r=1$ and $K+1$. Then showing $\left\|\mathbf{W}_{\mathcal{S}^{c} \mathcal{S}}^{-1} \mathbf{B W}_{\mathcal{S}, \mathcal{S}}\right\|_{\infty}<1$ is equivalent to proving

$$
g\left(s_{r-1}\right)+\frac{\left[g\left(s_{r}\right)-g\left(s_{r-1}\right)\right] z}{s_{r}-s_{r-1}}<g\left(s_{r-1}+z\right)
$$

for $2 \leq r \leq K$, where $z$ is a continuous variable such that $z \in\left[1, s_{r}-s_{r-1}-1\right]$. We know that $g\left(s_{r-1}+z\right)$ is a concave function with respect to $z$, whereas the left-hand side of (12) is affine in $z$. We also know that these two functions intersect at $z=0$ and $z=s_{r}-s_{r-1}$. Now the conclusion follows from the concavity of $g$.

Observe that $\nu=\left\|\mathbf{W}_{\mathcal{S}^{c}, \mathcal{S}^{c}}^{-1} \mathbf{B} \mathbf{W}_{\mathcal{S}, \mathcal{S}}\right\|_{\infty}$ determines the sensitivity of NFL to noise. This effect has been studied previously in the general framework of LASSO [14], [15]. In the next section, we study this sensitivity in detail to analyze the performance of NFL.

\section{PERformance ANALYsis OF NFL}

The next theorem is inspired by Lemma 3.4 in [14], and provides deterministic sufficient conditions under which NFL accurately detects change points.

Theorem 1. Assume that for a particular realization of $\tilde{\epsilon}$ there is a $\lambda_{p}>0$ such that

$$
\begin{aligned}
& \left\|\sigma \tilde{\mathbf{A}}_{\mathcal{S}^{c}}^{T} \Pi_{\mathcal{S}}^{\perp} \tilde{\boldsymbol{\epsilon}}+\lambda_{p} \tilde{\mathbf{A}}_{\mathcal{S}^{c}}^{T} \tilde{\mathbf{A}}_{\mathcal{S}}^{\dagger^{T}} \operatorname{sgn}\left(\tilde{\mathbf{x}}_{\mathcal{S}}^{*}\right)\right\|_{\infty}<\lambda_{p}, \\
& \min _{i \in \mathcal{S}}\left|\tilde{x}_{i}^{*}\right|>\left\|\sigma \tilde{\mathbf{A}}_{\mathcal{S}}^{\dagger} \tilde{\boldsymbol{\epsilon}}-\lambda_{p}\left(\tilde{\mathbf{A}}_{\mathcal{S}}^{T} \tilde{\mathbf{A}}_{\mathcal{S}}\right)^{-1} \operatorname{sgn}\left(\tilde{\mathbf{x}}_{\mathcal{S}}^{*}\right)\right\|_{\infty},
\end{aligned}
$$

where the matrix $\tilde{\mathbf{A}}_{\mathcal{S}}^{\dagger}=\left(\tilde{\mathbf{A}}_{\mathcal{S}}^{T} \tilde{\mathbf{A}}_{\mathcal{S}}\right)^{-1} \tilde{\mathbf{A}}_{\mathcal{S}}^{T}$ is the Moore-Penrose pseudo-inverse of $\tilde{\mathbf{A}}_{\mathcal{S}}$ and $\boldsymbol{\Pi}_{\mathcal{S}}^{\perp}$ denotes the orthogonal projection matrix defined as $\Pi_{\mathcal{S}}^{\perp}=\mathbf{I}-\tilde{\mathbf{A}}_{\mathcal{S}} \tilde{\mathbf{A}}_{\mathcal{S}}^{\dagger}$. Then, $\hat{\mathbf{x}}^{\mathrm{NFL}}$, obtained by solving (10) with $\lambda=\lambda_{p}$, satisfies $\operatorname{supp}\left(\hat{\mathbf{x}}^{\mathrm{NFL}}\right)=\mathcal{S}$ and $\operatorname{sgn}\left(\hat{\mathbf{x}}_{\mathcal{S}}^{\mathrm{NFL}}\right)=\operatorname{sgn}\left(\tilde{\mathbf{x}}_{\mathcal{S}}^{*}\right)$.

Proof. From the optimality conditions for (10),we know that

$$
\left\|\tilde{\mathbf{A}}_{\mathcal{S}^{c}}^{T}\left(\tilde{\mathbf{y}}-\tilde{\mathbf{A}} \hat{\mathbf{x}}^{\mathrm{NFL}}\right)\right\|_{\infty}<\lambda_{p}
$$

ensures $\hat{\mathbf{x}}_{\mathcal{S}^{c}}^{\mathrm{NFL}}=\mathbf{0}$ (see, e.g., Lemma 2.1 in [18]). Hence, under this condition we can write $\hat{\mathbf{x}}^{\mathrm{NFL}}=\tilde{\mathbf{x}}^{*}+\mathbf{r}$ for some $\mathbf{r}$ with $\mathbf{r}_{\mathcal{S}^{c}}=\mathbf{0}$. Now if

$$
\min _{i \in \mathcal{S}}\left|\tilde{x}_{i}^{*}\right|>\left\|\mathbf{r}_{\mathcal{S}}\right\|_{\infty}
$$

then we have $\operatorname{supp}\left(\hat{\mathbf{x}}^{\mathrm{NFL}}\right)=\mathcal{S}$ and $\operatorname{sgn}\left(\hat{\mathbf{x}}_{\mathcal{S}}^{\mathrm{NFL}}\right)=\operatorname{sgn}\left(\tilde{\mathbf{x}}_{\mathcal{S}}^{*}\right)$. From the optimality conditions of (10), we must also have

$$
\tilde{\mathbf{A}}_{\mathcal{S}}^{T}\left(\tilde{\mathbf{y}}-\tilde{\mathbf{A}} \hat{\mathbf{x}}^{\mathrm{NFL}}\right)=\lambda_{p} \operatorname{sgn}\left(\hat{\mathbf{x}}_{\mathcal{S}}^{\mathrm{NFL}}\right),
$$

which can be rewritten as

$$
\tilde{\mathbf{A}}_{\mathcal{S}}^{T}\left(\tilde{\mathbf{A}}_{\mathcal{S}} \tilde{\mathbf{x}}_{\mathcal{S}}^{*}+\sigma \tilde{\boldsymbol{\epsilon}}-\tilde{\mathbf{A}}_{\mathcal{S}}\left(\tilde{\mathbf{x}}_{\mathcal{S}}^{*}-\mathbf{r}_{\mathcal{S}}\right)\right)=\lambda_{p} \operatorname{sgn}\left(\hat{\mathbf{x}}_{\mathcal{S}}^{\mathrm{NFL}}\right) .
$$

Solving the preceding equation for $\mathbf{r}_{\mathcal{S}}$ gives $\mathbf{r}_{\mathcal{S}}=$ $\left(\tilde{\mathbf{A}}_{\mathcal{S}}^{T} \tilde{\mathbf{A}}_{\mathcal{S}}\right)^{-1}\left[\sigma \tilde{\mathbf{A}}_{\mathcal{S}}^{T} \tilde{\boldsymbol{\epsilon}}-\lambda_{p} \operatorname{sgn}\left(\tilde{\mathbf{x}}_{\mathcal{S}}^{*}\right)\right]$. Finally, inserting the explicit expressions for $\tilde{\mathbf{y}}$ and $\hat{\mathbf{x}}^{\mathrm{NFL}}$ into (15) and (16) yields (13) and (14), respectively.

Note that when $\tilde{\epsilon}$ is a random vector, (13) holds with high probability for a proper choice of $\lambda_{p}$ [14]. Therefore, if $\min _{i \in \mathcal{S}}\left|\tilde{x}_{i}^{*}\right|$ is large enough, NFL most likely detects the true change points. Furthermore, as $\sigma \rightarrow 0$, (13) simplifies to the irrepresentable condition, which was shown to hold in Lemma 1. Relating to (14), using norm properties, we can say

$$
\begin{aligned}
\left\|\left(\tilde{\mathbf{A}}_{\mathcal{S}}^{T} \tilde{\mathbf{A}}_{\mathcal{S}}\right)^{-1} \operatorname{sgn}\left(\tilde{\mathbf{x}}_{\mathcal{S}}^{*}\right)\right\|_{\infty} & \leq \sqrt{K}\left\|\left(\tilde{\mathbf{A}}_{\mathcal{S}}^{T} \tilde{\mathbf{A}}_{\mathcal{S}}\right)^{-1}\right\|_{2} \\
& =\frac{\sqrt{K}}{\Lambda_{\min }\left(\tilde{\mathbf{A}}_{\mathcal{S}}^{T} \tilde{\mathbf{A}}_{\mathcal{S}}\right)},
\end{aligned}
$$

where $\Lambda_{\min }(\cdot)$ represents the smallest eigenvalue. Therefore, if $\min _{i \in \mathcal{S}}\left|\tilde{x}_{i}^{*}\right| \Lambda_{\min }\left(\tilde{\mathbf{A}}_{\mathcal{S}}^{T} \tilde{\mathbf{A}}_{\mathcal{S}}\right)>\lambda_{p} \sqrt{K}$, NFL is asymptotically consistent in detecting $\mathcal{S}$; i.e., $\operatorname{Pr}\left(\operatorname{supp}\left(\hat{\mathbf{x}}^{\mathrm{NFL}}\right)=\mathcal{S}\right) \rightarrow 1$ as $\sigma \rightarrow 0$.

\section{EMPIRICAL RESULTS}

In this section, we provide numerical experiments to illustrate the performance of NFL. The performance of NFL is compared with those of FL and iterative penalized FL (IPFL) [12]. IPFL is an iterative method that solves a weighted FL within successive iterations. The weighted FL in iteration $l$ can be formulated as

$$
\hat{\mathbf{m}}^{\mathrm{IP}}(l+1)=\underset{\mathbf{m} \in \mathbb{R}^{N}}{\operatorname{argmin}}\left\{\frac{1}{2}\|\mathbf{y}-\mathbf{m}\|_{2}^{2}+\lambda\|\mathbf{h}(l) \odot(\mathbf{D m})\|_{1}\right\},
$$

where $\odot$ denotes elementwise multiplication and the elements of the weight vector $\mathbf{h}(l)$ are defined as

$$
h_{i}(l)=\exp \left(-\left|\left[\mathbf{D} \hat{\mathbf{m}}^{\mathrm{IP}}(l)\right]_{i}\right| / \varrho\right)
$$

and $\varrho>0$ is a constant. It is suggested to set the parameter $\varrho$ to $4 \lambda$ [12].

In all considered methods FL, NFL and IPFL, the parameter $\lambda$ is known to control the support of the solution [12], [19], [20], and it has to be tuned appropriately. For instance, $\operatorname{supp}\left(\hat{\mathbf{x}}^{\mathrm{NFL}}\right)$ evolves as $\lambda$ varies from $\left\|\tilde{\mathbf{A}}^{T} \mathbf{y}\right\|_{\infty}$ down to zero, and typically, the cardinality of $\operatorname{supp}\left(\hat{\mathbf{x}}^{\mathrm{NFL}}\right)$ is a decreasing function of $\lambda$. Ideally, we would like to select $\lambda$ satisfying the conditions of Theorem 1, so that the NFL estimator returns the true support. Many approaches have been proposed in the literature to tune $\lambda$. In [14] and [21], $\lambda$ is chosen of the order 


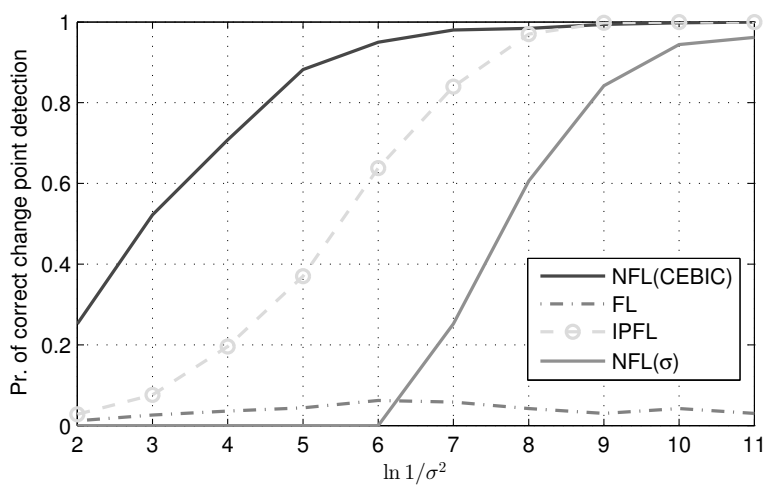

Fig. 2. The probability of correct change point detection versus $\ln \left(1 / \sigma^{2}\right)$ when $N=250$ and $\left[\bar{m}_{1}, \bar{m}_{2}, \bar{m}_{3}\right]=[1,2,3]$.

of $\sigma \sqrt{2 \ln N}$. This choice requires the knowledge of $\sigma$, and yields a detector whose performance only slowly increases with the number of samples $N$. Hence, to overcome these issues in tuning $\lambda$ for NFL and FL, we adopt the recently proposed model selection approach, CEBIC [22]. CEBIC in principle takes the set of evolution of $\left\{\operatorname{supp}\left(\hat{\mathbf{x}}^{\mathrm{NFL}}\right), \lambda\right\}$ from the LASSO's solution-path (i.e. solving LASSO piecewise linearly [20]) and finds which $\operatorname{supp}\left(\hat{\mathbf{x}}^{\mathrm{NFL}}\right)$ describes data the best. Unfortunately, due to the complicated nature of IPFL, it is not possible to find its solution-path with respect to $\lambda$. Therefore, for IPFL we set $\lambda$ to $4 \sigma \sqrt{N}$, as suggested in [12] (as if $\sigma$ were known).

Now we first provide a numerical experiment to support our theoretical claim. To do so, the probability of correctly detecting the change points is measured versus $\ln \left(1 / \sigma^{2}\right)$ over 500 Monte Carlo trials when $N$ is fixed to 250. In this numerical experiment, the number of change points is fixed to two and the true amplitudes are $\left[\bar{m}_{1}, \bar{m}_{2}, \bar{m}_{3}\right]=[1,2,3]$. This means that the experiment only focuses on $\operatorname{sgn}\left(x_{s_{1}}^{*}\right)=\operatorname{sgn}\left(x_{s_{2}}^{*}\right)=1$. The true change points are specified in each Monte Carlo trial randomly, such that (2) is satisfied. The random perturbation is a white Gaussian noise with $\epsilon \sim \mathcal{N}(0,1)$. Fig. 2 illustrates the empirical probability of correct change point detection versus $\ln \left(1 / \sigma^{2}\right)$ under the aforementioned setting. The numerical experiment shows that NFL detects the change points perfectly if the perturbation is sufficiently small. As can be seen, NFL with properly tuned $\lambda$, NFL(CEBIC), outperforms IPFL. NFL $(\sigma)$ illustrates the performance of NFL when $\lambda$ is selected of the order of $\sigma \sqrt{2 \ln N}$. The huge gap between $\operatorname{NFL}(\mathrm{CEBIC})$ and $\operatorname{NFL}(\sigma)$ shows the sensitivity of LASSO to the tuning parameter. This experiment also shows that FL completely fails in exactly detecting $\mathcal{S}$, as was expected from the discussion in Section II-B.

Next, we consider the effect of the temporal distance of $s_{i}$ 's on the accuracy of detection. In this regard, the probability of correctly detecting the change points is measured versus $s_{2}-s_{1}$ over 500 Monte Carlo trials for $N=200$ and $\sigma=0.1$. Through the experiment, the change points gradually move apart according to $s_{1}=99-\Delta / 2$ and $s_{2}=101+\Delta / 2$, where the initial value of $\Delta$ is 10 . It is expected that a small temporal distance between $s_{i}$ 's decreases the accuracy of detection. This anticipation is due to the structure of $\tilde{\mathbf{A}}$. Roughly speaking, $\tilde{\mathbf{A}}$

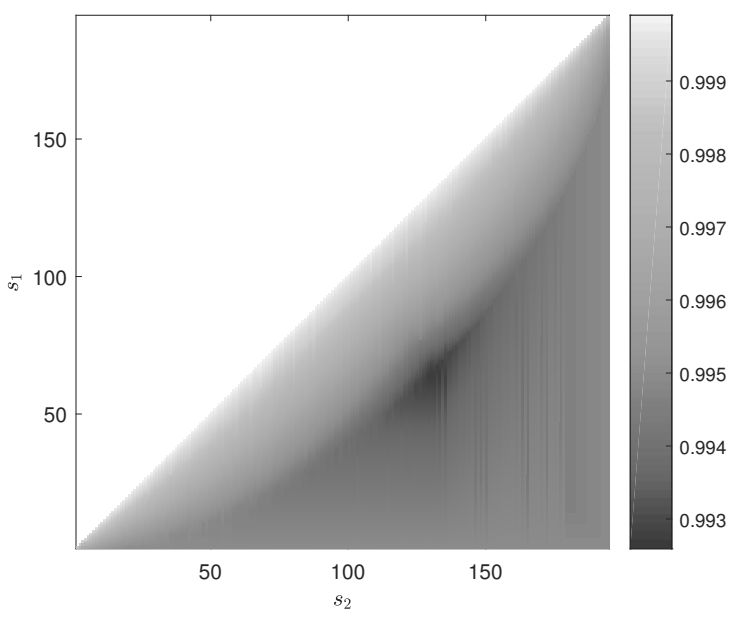

Fig. 3. Graphical illustration of $\nu$ as a function of $\mathcal{S}$ when $K=2$ and $N=200$.

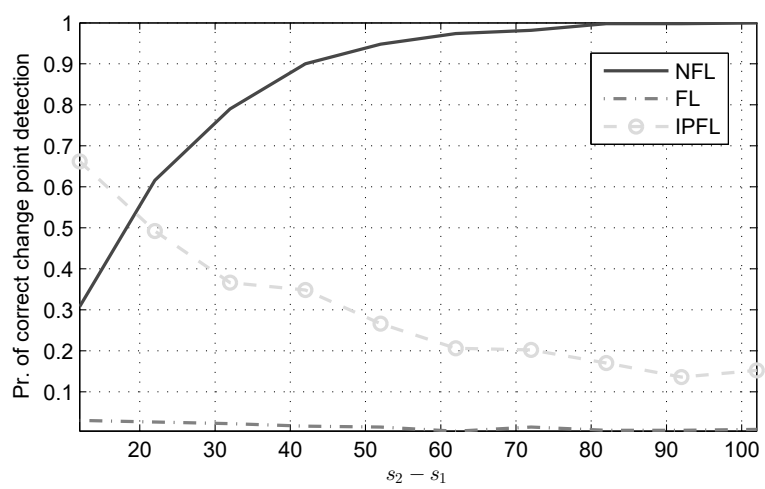

Fig. 4. The probability of correct change point detection versus $s_{2}-s_{1}$ when $\sigma=0.1, N=200$ and $\left[\bar{m}_{1}, \bar{m}_{2}, \bar{m}_{3}\right]=[1,2,3]$.

is structured such that the distance between columns, $|i-j|$, affects $\nu$ inversely. See Fig. 3. Therefore, by considering noise, NFL has little chance in finding $s_{i}$ 's associated with a small temporal distance. Fig. 4 depicts the probability of correct change point detection versus $s_{2}-s_{1}$. The probability of $\operatorname{supp}\left(\hat{\mathbf{x}}^{\mathrm{NFL}}\right)=\mathcal{S}$ for NFL converges to one as $s_{2}-s_{1}$ increases. In contrast, this probability for IPFL decreases as $s_{2}-s_{1}$ increases. This is perhaps related to how the choice of $\lambda$ and $\varrho$ affects the performance of IPFL. For the same reasons as before, FL shows poor performance in detecting $s_{i}$ 's.

\section{CONCLUSION}

It is known that FL is inconsistent in detecting the true change points from a noisy piecewise constant signal. To overcome this issue, we have proposed a new method, NFL. Our method is indeed nothing but a normalized formulation of FL. We prove that NFL satisfies the irrepresentable condition. This implies that, as $\sigma \rightarrow 0$ our method consistently detects the true change points. The numerical results confirm that NFL detects the change points perfectly when the perturbation is small enough. Additionally, we highlight that NFL and FL have the same computational complexity. 


\section{REFERENCES}

[1] N. R. Zhang and D. O. Siegmund, "A modified bayes information criterion with applications to the analysis of comparative genomic hybridization data," Biometrics, vol. 63, no. 1, pp. 22-32, 2007.

[2] E. Keogh, K. Chakrabarti, M. Pazzani, and S. Mehrotra, "Locally adaptive dimensionality reduction for indexing large time series databases," ACM SIGMOD Record, vol. 30, no. 2, pp. 151-162, 2001.

[3] Y. Zhang, M. Brady, and S. Smith, "Segmentation of brain mr images through a hidden markov random field model and the expectationmaximization algorithm," IEEE Trans. Med. Imaging, vol. 20, no. 1, pp. 45-57, Jan. 2001.

[4] K. C. Sou, J. Weimer, H. Sandberg, and K. H. Johansson, "Scheduling smart home appliances using mixed integer linear programming," in IEEE 50th Conf. Decision and Control and European Control Conf., 2011, pp. 5144-5149.

[5] L. I. Rudin, S. Osher, and E. Fatemi, "Nonlinear total variation based noise removal algorithms," Physica D: Nonlinear Phenomena, vol. 60, no. 1, pp. 259-268, 1992.

[6] C. Levy-leduc and Z. Harchaoui, "Catching change-points with lasso," in Adv. Neural. Inf. Process. Syst., 2008, pp. 617-624.

[7] R. Tibshirani, M. Saunders, S. Rosset, J. Zhu, and K. Knight, "Sparsity and smoothness via the fused lasso," J. Roy. Statisti. Soc.: Series B (Statistical Methodology), vol. 67, no. 1, pp. 91-108, 2005.

[8] M. A. Little and N. S. Jones, "Generalized methods and solvers for noise removal from piecewise constant signals. i. background theory," Proc. Royal Society, vol. 467, no. 2135, pp. 3088-3114, 2011.

[9] R. Tibshirani, "Regression shrinkage and selection via the lasso," $J$. Roy. Statisti. Soc. Series B (Methodological), vol. 58, no. 1, pp. 267288,1996

[10] J. Ottersten, B. Wahlberg, and C. R. Rojas, "Accurate changing point detection for mean filtering," IEEE Signal Process. Lett., vol. 23, no. 2, pp. 297-301, Feb. 2016.

[11] C. R. Rojas and B. Wahlberg, "On change point detection using the fused Lasso method," ArXiv:1401.5408, Jan. 2014

[12] M. Malek-Mohammadi, R. C. Rojas, and B. Wahlberg, "A class of nonconvex penalties preserving overall convexity in optimization-based mean filtering," IEEE Tran. Signal Process., vol. 64, no. 24, pp. 66506664, Dec. 2016

[13] P. Zhao and B. Yu, "On model selection consistency of lasso," J. Mach. Learn. Res., vol. 7, pp. 2541-2563, Nov. 2006.

[14] E. Candes and Y. Plan, "Near-ideal model selection by $\ell_{1}$ minimization," Ann. Statist., vol. 37, no. 5A, pp. 2145-2177, Oct. 2009.

[15] M. J. Wainwright, "Sharp thresholds for high-dimensional and noisy sparsity recovery using $\ell_{1}$-constrained quadratic programming (lasso), IEEE Trans. Inf. Theory, vol. 55, no. 5, pp. 2183-2202, May 2009.

[16] Á. Barbero and S. Sra, "Modular proximal optimization for multidimensional total-variation regularization," arXiv preprint arXiv:1411.0589, 2014.

[17] L. Condat, "A direct algorithm for $1 \mathrm{~d}$ total variation denoising," IEEE Signal Process. Lett., vol. 20, no. 11, pp. 1054-1057, Nov. 2013.

[18] P. Bühlmann and S. Van De Geer, Statistics for high-dimensional data: methods, theory and applications, Springer, 2011.

[19] R. J. Tibshirani and J. Taylor, "The solution path of the generalized lasso," Ann. Statist., vol. 39, no. 3, pp. 1335-1371, Jun. 2011.

[20] B. Efron, T. Hastie, I. Johnstone, and R. Tibshirani, "Least angle regression," Ann. Statist., vol. 32, no. 2, pp. 407-499, Apr. 2004.

[21] S. Chretien and S. Darses, "Sparse recovery with unknown variance: A lasso-type approach," IEEE Tran. Inf. Theory, vol. 60, no. 7, pp. 3970-3988, July 2014.

[22] A. Owrang and M. Jansson, "Model selection for high-dimensional data," IEEE Asilomar Conf. on Signals, Systems and Computers, 2016. 